\title{
Future growth and success of nuclear cardiology
}

\author{
George A. Beller, MD \\ a Division of Cardiovascular Medicine, University of Virginia Health System, Charlottesville, VA
}

Received Jan 22, 2018; accepted Jan 22, 2018

doi: $10.1007 / \mathrm{s} 12350-018-1211-1$

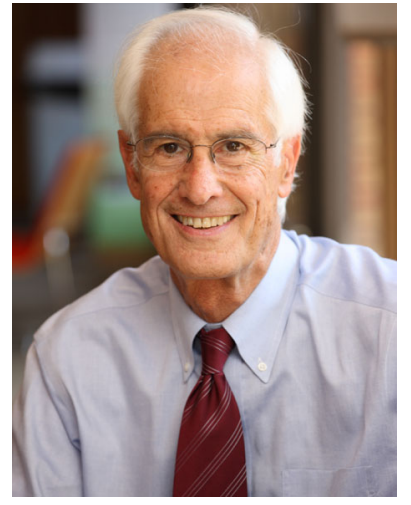

George A. Beller,

Editor Emeritus,

Journal of Nuclear Cardiology

About 6 months ago, I reviewed a manuscript, and subsequently wrote an editorial, ${ }^{1}$ pertaining to a retrospective study undertaken by Mayo Clinic investigators, reporting a marked decline in the annual volume of SPECT myocardial perfusion imaging (MPI) studies in patients with known CAD, from 1425 in 2003, to 552 in $2012 .^{2}$ This report followed a prior publication from the same group in 2016, indicating that the volume of SPECT MPI in patients without known CAD had decreased from 2490 in 2003 to 1033, in $2012 .^{3}$ In the more recent report, ${ }^{2}$ the authors further showed that the percentage of high-risk SPECT results also diminished over time, from $47.8 \%$ of all studies in years 1991-1995, to only $8.1 \%$ in years 2011-2012. This decrease in highrisk SPECT imaging results occurred, even though the prevalence of CAD risk factors, except for smoking, in the tested population increased over this time period. A similar observation was made by the Cedars Sinai group, ${ }^{4}$ which reported a progressive decrease in abnormal SPECT MPI studies from 40.9\% in 1991 to $8.7 \%$ in 2009 . The incidence of stress-induced ischemia fell from $29.6 \%$ to $5.0 \%$ of imaging studies,

Reprint requests: George A. Beller, MD, Division of Cardiovascular Medicine, University of Virginia Health System, Box 800158, Charlottesville, VA 22903; GAB4C@hscmail.mcc.virginia.edu J Nucl Cardiol 2018;25:375-8.

$1071-3581 / \$ 34.00$

Copyright (C) 2018 American Society of Nuclear Cardiology. accompanied by a marked decrease in patients manifesting moderate-to-severe ischemia.

Other studies in the literature confirm the Mayo Clinic observation of a decrease in volume of SPECT MPI in the past decade. ${ }^{5,6}$ Medicare data revealed that the radionuclide MPI rate per 100 Medicare beneficiaries rose from 63.4 in 2001, to a high of 88.0 in 2006, but then significantly declined each year thereafter, reaching a value of 10.8 in 2013. ${ }^{5}$ A Kaiser Permanente database study revealed a $51 \%$ decline in the annual rate of MPI procedures from 2006 to $2011 .{ }^{6}$ Other reports in the literature also confirm the diminution over time of abnormal test results. ${ }^{7,8}$ The average rate of abnormal stress tests in 4 multicenter studies in which functional stress testing was compared to coronary computed tomography angiography (CTA) was only $14.5 \% .^{8}$ The Prospective Multicenter Imaging Study for Evaluation of Chest Pain (PROMISE) trial showed that just 12.6\% of patients in the functional testing arm had abnormal tests. ${ }^{9}$ The risk of death, nonfatal myocardial infarction, hospitalization for unstable angina, or major complications related to diagnostic testing or cardiovascular procedures after an abnormal functional test was only $3.0 \%$ at 25 months of follow-up. ${ }^{9}$ These data showed that the prognosis in the population tested was quite favorable, even after a positive test result. At the University of Virginia, a total of 3302 SPECT MPI studies, and 347 gated blood pool scan (MUGA) studies, were performed in 2006. By 2017, the number of SPECT MPI and MUGA procedures had decreased to 2021 and 103, respectively. However, this decrease in SPECT MPI was attenuated by the performance of 365 PET MPI studies in 2017.

The possible explanations for the decrease in volume in SPECT MPI and fewer high-risk imaging findings are myriad, and were discussed in the editorial. ${ }^{1}$ Certainly, the emergence of Appropriate Use Criteria in 2005 had an impact on reducing referrals for SPECT MPI, particularly in asymptomatic patients, and those that had undergone uncomplicated revascularization procedures. Routine functional stress testing after an uncomplicated acute coronary syndrome, or 3-6 months 
after percutaneous coronary intervention (PCI) or coronary artery bypass surgery (CABG), was deemed to be not indicated in AUC or practice guidelines. Barriers to SPECT MPI were erected by insurance companies with radiology benefits' managers requiring pre-authorization for testing. It is possible that more patients presenting with typical angina were being referred directly to invasive coronary angiography. Similarly, in recent years, some patients are referred for CTA rather than functional stress testing for the noninvasive evaluation of chest pain. It is likely that more patients with atypical symptoms and good exercise tolerance are undergoing exercise stress testing without imaging as the first diagnostic test. Because of more attention being paid to the radiation exposure of diagnostic imaging, perhaps, fewer young adults, particularly women, are being referred for stress radionuclide imaging for testing.

Fewer high-risk stress MPI results in imaging are most likely contributed to by better treatment of CAD risk factors and a decrease in smoking in the population. Diabetic patients without overt heart disease are being place on cholesterol-lowering drugs, resulting in fewer high-risk SPECT MPI findings associated with better outcomes even after an abnormal scan. ${ }^{10}$ The overall observed reduction in the incidence of stress-induced ischemia parallels the improved prognosis of patients on optimal medical therapy, including statins and effective blood pressure control.

For almost 40 years, the field of nuclear cardiology has been dominated by perfusion imaging. Early in the development of the field, enthusiasm was expressed for infarct imaging with Tc-99m-pyrophosphate. This dissipated with the emergence of biomarker testing for detection of infarction in its early stages. First pass and equilibrium gated cardiac blood pool imaging were nuclear cardiology technologies that yielded accurate measurement of left ventricular ejection fraction and assessment of regional wall motion. One of these blood pool imaging tests was often utilized for monitoring the potential cardiac complications of cancer chemotherapy. However, these radionuclide angiographic procedures have also markedly declined in usage, in favor of echocardiography and cardiac magnetic resonance (MRI).

It is clear that thought leaders in the field of nuclear cardiology, including the leadership of the American Society of Nuclear Cardiology (ASNC), became concerned with this declining volume of SPECT MPI, and the need to provide relevant imaging strategies that demonstrate their worth in a value-based healthcare system. In June 2017, the ASNC Board of Directors convened a meeting with industry representatives and other stakeholders to "redefine the value of nuclear cardiology and the priorities for future growth and success. ${ }^{11,}$, The ASNC board and their industry partners proposed some "solutions-based" initiatives and projects that were judged to propel the field of nuclear cardiology into the future. These recommendations were focused on the delivery of high-quality appropriate imaging, promoting the unique benefits of cardiac PET, modernizing the practice of cardiac SPECT, promoting nonperfusion applications of nuclear cardiology, and developing a road map to high-quality imaging. Certainly, advocating for high-quality appropriate imaging and modernizing SPECT practice are of paramount importance, but this alone may not have a great impact on expanding the field of nuclear cardiology with a concomitant increase in volume and diversity of procedures. Nevertheless, old SPECT cameras need to be replaced by more sensitive solid state cameras that permit lower doses of tracers without sacrificing quality. Similarly, it appears that more and more practitioners are adhering to AUC indications for imaging, with fewer inappropriate tests being ordered.

What will truly make a difference in expanding nuclear cardiology in the future are the Industry Forum recommendations related to advancing cardiac PET and developing new nonperfusion SPECT and PET applications. The validation of these nonperfusion imaging protocols is currently underway in the form of feasibility studies, reports of small patient series, and carrying out of outcomes studies to show their clinical value in patient diagnosis and management. PET MPI has a better resolution, a higher sensitivity, and specificity for detection of CAD; is superior for detecting multivessel disease; and has lower radiation exposure than SPECT MPI. ${ }^{12}$ It allows for quantitative measurement of myocardial blood coronary flow and coronary flow reserve (CFR) using vasodilator stress. Measurement of CFR is associated with improved detection of multivessel disease and quantitating abnormal flow reserve improves risk stratification compared to solely assessing relative defects in tracer uptake. Abnormal CFR can be seen in patients with nonobstructive CAD and patients with microvascular dysfunction. These patients without focal defects due to significant obstructive CAD, but abnormal CFR have a worse prognosis than patients with normal CFR and no inducible defects. Although some nuclear cardiology laboratories have performed more PET MPI studies in recent years, volumes are limited by access to the instrumentation, which is often shared with users in other specialties such as oncology. Nuclear cardiology laboratories require dedicated PET cameras to provide a better access to cameras. Many of the nonperfusion applications of PET would also benefit from enhanced access to PET instrumentation. Hybrid imaging with PET/CT provides even greater information pertaining to anatomy and physiology simultaneously 
assessed. The Industry Forum report identifies the need for educating third-party payers on the value of cardiac PET imaging, which was deemed essential to helping break down the barriers to access for PET. Presently, the MPI radiotracer, F-18-flurpiridaz, is in Phase III trials. This long-acting PET tracer with excellent myocardial extraction can be utilized in exercise PET MPI, and has already been shown to have accuracy superior to SPECT in Phase II trials. ${ }^{12}$

The acceptance and growth of nonperfusion cardiac imaging applications are vital in widening the offerings of nuclear cardiology going forward. Already, much activity has been ongoing in validating the worth of F18-flurodeoxyglucose (FDG) imaging for detecting and quantitating focal inflammatory lesions in patients with sarcoidosis. $^{13,14}$ From 8 published studies, the pooled sensitivity and specificity values for detecting cardiac involvement with sarcoid were $89 \%$ and $78 \%$, respectively. Combining MPI with FDG imaging could even enhance the accuracy of detection of sarcoid granulomas in the heart. Even combining PET with MRI for showing areas of delayed hyperenhancement in areas of decreased perfusion without inflammation could be an early manifestation of cardiac sarcoid. ${ }^{15}$

Hybrid imaging with PET/CT or SPECT/CT is being evaluated for detection of cardiovascular implant infection including surgically placed valvular prostheses, transcatheter aortic valve implantations (TAVI), pacemakers, pacemaker leads, implantable defibrillators, and left ventricular assist devices. ${ }^{16}$ The clinical value of neurocardiac imaging of the sympathetic nervous system with SPECT or PET continues to be evaluated in clinical trials. ${ }^{17,18}$ A new trial of I-123-metaiodobenzylguanidine (MIBG) imaging involves randomizing patients with LVEF values between $30 \%$ and $35 \%$ (ADMIREICD) to determine if MIBG imaging useful for guiding the decision for ICD implantation. This trial is scheduled to be completed in 2019. Tc-99m-pyrophosphate (PYP) imaging can be used for detection of transthyretin cardiac amyloidosis. ${ }^{19}$ F-18-Florbetapir binds to cardiac amyloid light-chain and transthyretin-related amyloid deposits. $^{20}$ This PET approach to early detection of this pathology could be useful for more prompt treatment before progression of the disease. Finally, work is continuing to explore the clinical value in decision-making for imaging vulnerable atherosclerotic plaques which are inflamed. ${ }^{21}$ Atherosclerotic plaque uptake of FDG correlates with macrophage infiltration, and increased plaque FDG uptake in the arterial wall may identify a higher risk for acute cardiac events. F-18-fluoride targets microcalcifications in the fibrous caps of atherosclerotic plaques. $^{22}$ So far, plaque imaging has been found to have a low positive predictive value but an excellent negative predictive value for excluding lesions leading to events. ${ }^{22}$ As with other nonperfusion imaging studies under investigation, evidence for clinical value lies in the ability of the imaging technique to predict hard clinical endpoints and influencing outcomes.

In conclusion, the future growth and progress in nuclear cardiology will include greater utilization of PET for quantitative MPI with CFR measurement, and for metabolic and molecular nonperfusion imaging applications. This can be accomplished while still adhering to the delivery of high-quality and appropriate SPECT myocardial perfusion imaging, but with better cameras and software applications. The use of multimodality imaging in certain disease states will surely provide even greater information that can yield an early diagnosis, direct the most effective evidence-based therapy, and improve outcomes. For nuclear cardiology to grow and thrive, robust basic and clinical research strategies are required for identifying new biologic tracers for specific diseases, which, when translated to clinical imaging protocols, will have an impact in patient outcomes that can be measured. For too many years, nuclear cardiology has been dependent on perfusion imaging, which certainly has been of great clinical value in the evaluation of patients with suspected or known CAD. It is exciting to see that currently, many innovative nonperfusion imaging procedures are being evaluated in clinical research studies. Some are in randomized trials. The ASNC Board of Directors with its Industry partners and other stakeholders are diligently working to identify a new road map to redefine the value of nuclear cardiology, with support of new emerging applications and innovations to enhance the field. Personally, as someone who has witnessed the progress of nuclear cardiology over more than 40 years, I remain optimistic for the future. This optimism is predicated on the assumption that we continue to attract the next generation of academic imaging specialists to move the field forward by engaging in translational research to find new applications of nuclear cardiology that have a positive impact on patient outcomes.

\section{Disclosure}

The author declare that he has no conflict of interest.

\section{References}

1. Beller GA. How should we interpret the decrease in annual volume of stress imaging tests for evaluation of suspected or known coronary artery disease with fewer high-risk tests? Circ Cardiovasc Imaging. 2017. https://doi.org/10.1161/CIRCIMAGING.117. 006702 . 
2. Jouni H, Askew JW, Crusan DJ, Miller TD, Gibbons RF. Temporal trends of single-photon emission computed tomography myocardial perfusion imaging in patients with coronary artery disease: A 22-year experience from a tertiary academic medical center. Circ Cardiovasc Imaging. 2017. https://doi.org/10.1161/ CIRCIMAGING.117.006702.

3. Jouni H, Askew JW, Crusan DJ, Miller TD. Gibbons Rj. Temporal trends of single-photon emission computed tomography myocardial perfusion imaging in patients without prior coronary artery disease: a 22-year experience at a tertiary academic medical center. Am Heart J. 2016;176:27-133.

4. Rozanski A, Gransar H, Hayes SW, Min J, Friedman JD, Thomson LE, et al. Temporal trends in the frequency of inducible myocardial ischemia during cardiac stress testing: 1991 to 2009. J Am Coll Cardiol. 2013;2013(61):1054-65.

5. Levin DC, Parker L, Halpern EJ, Rao VM. Recent trends in imaging for suspected coronary artery disease: what is the best approach? J Am Coll Radiol. 2016;13:381-6.

6. McNulty EJ, Hung YY, Almers LM, Go AS, Yeh RW. Population trends from 2000-2011 in nuclear myocardial perfusion imaging use. JAMA. 2014;311:1248-9.

7. Duvall WL, Rai M, Ahlberg AW, O'Sullivan DM, Henzlova MJ. A multicenter assessment of the temporal trends in myocardial perfusion imaging. J Nucl Cardiol. 2015;22:539-51.

8. Shaw LJ, Phillips LM, Nagel E, Newby DE, Narula J, Douglas PS. Comparative effectiveness trials of imaging-guided strategies in stable ischemic heart disease. J Am Coll Cardiol Img. 2017;10: $321-4$.

9. Douglas PS, Hoffmann U, Patel MR, Mark DB, Al-Khalidi HR, Cavanaugh B, et al. Outcomes of anatomical versus functional testing for coronary artery disease. N Engl J Med. 2015;372:1291300.

10. Bourque JM, Patel CA, Ali MM, Perez M, Watson DD, Beller GA. Prevalence and predictors of ischemia and outcomes in outpatients with diabetes mellitus referred for single-photon emission computed tomography perfusion imaging. Circ Cardiovasc Imaging. 2013;6:466-77.

11. ASNC 2017 Industry Forum Report.

12. Maddahi J, Packard RRS. PET should replace SPECT in cardiac imaging for diagnosis and risk assessment of patients with known or suspected CAD: Pro. J Nucl Cardiol. 2017;24:1955-9.
13. Chareonthaitawee P, Beanlands RS, Chen W, Dorbala S, Miller EJ, Murthy VL, et al. Joint SNMMI-ASNC expert consensus document on the role of F-18-FDG PET/CT in cardiac sarcoid detection and therapy monitoring. J Nucl Cardiol. 2017;24:174158.

14. Di Carli MF, Geva T, Davidoff R. The future of cardiovascular imaging. Circulation. 2016;133:2640-61.

15. Schindler TH. Cardiovascular PET/MR imaging: Quo Vadis? J Nucl Cardiol. 2017;24:1007-18.

16. Sohns JM, Bavendiek U, Ross TL, Bengel FM. Targeting cardiovascular implant infection. Multimodality and molecular imaging. Circ Cardiovasc Imaging. 2017;10:e005376. https://doi.org/10.1161/ CIRCIMAGING.117.005376.

17. Jacobson AF, Senior R, Cerqueira MD, Wong ND, Thomas GS, Lopez VA, et al. Myocardial iodine-123 meta-iodobenzylguanidine imaging and cardiac events in heart failure. Results of the prospective ADMIRE-HF (AdreView Myocardial Imaging for Risk Evaluation in Heart Failure) study. J Am Coll Cardiol. 2010;55:2212-21.

18. Nakata T, Nakajima K, Yamashina S, Yamada T, Momose M, Kasama S, et al. A pooled analysis of multicenter cohort studies of I-123-mIBG cardiac sympathetic innervation imaging for assessment of long-term prognosis in chronic heart failure. J Am Coll Cardiol Img. 2013;6:772-84.

19. Bokhari S, Castano A, Pozniakoff T, Deslisle S, Latif F, Maurer MS. (99m)Tc-pyrophosphate scintigraphy for differentiating lightchain cardiac amyloidosis from the transthyretin-related familial and senile cardiac amyloidosis. Circ Cardiovasc Imaging. 2013;6:195-201.

20. Park MA, Padera RF, Belanger A, Dubey S, Hwang DH, Veeranna $\mathrm{V}$, et al. 18F-Florbetapir binds specifically to myocardial lightchain and transthyretin amyloid deposits: Auto-radiography study. Circ Cardiovas Imaging. 2015. https://doi.org/10.1161/CIRCI MAGING.114.002954

21. Hammad B, Evans NR, Rudd JHF, Tawakol A. Molecular imaging of atherosclerosis with integrated PET imaging. J Nucl Cardiol. 2017;24:938-43.

22. Strauss HW, Narula J. 18F-fluoride imaging and other plaque-seeking diagnostic strategies: Understanding the reality and setting the ground rules. Circ Cardiovasc Imaging. 2017. https://doi.org/10.1161/CIRCI MAGING.117.007110. 